March 2018

\title{
"The Differentiated Effect of Advertising on Readership: Evidence from a Two-Sided Market Approach"
}

Marc Ivaldi, Catherine Muller-Vibes 


\title{
The Differentiated Effect of Advertising on Readership: Evidence from a Two-Sided Market Approach
}

\author{
Marc Ivaldi, \\ Toulouse School of Economics, \\ marc.ivaldi@tse-fr.eu \\ Catherine Muller-Vibes, \\ Université de Toulouse, Toulouse Business School, \\ catherine.muller@,tbs-education.fr
}

March 2018

\begin{abstract}
In this paper, we empirically analyze the French print media market by modeling the existence of a reciprocal effect between the size of the readership and the amount of advertising. For this two-sided platform, we measure the cross-effects of advertising on the readership and periodical popularity on advertising. By estimating a structural model of simultaneous demand equations, we quantify some crucial elements in designing pricing and product-differentiating strategies. We measure the impact of advertising on reader demand and find in the data that it has opposite effects depending on whether the publication presents informational or entertaining content. By taking into account the market interactions, we compute price and advertising elasticities. Our results show that advertisers targeting a specific category of the audience would choose its corresponding periodicals and would trade off the size of the readership for these periodicals and the advertising insert price changes. Also, advertising campaigns aimed at reaching a broader spectrum of the population should focus on popular titles and on titles for which demand is inelastic to ensure a more consistent impact of the campaign. Finally, for magazines with low price demand elasticity on the readers' side, editors' revenues could be improved by increasing prices. These combined effects should allow a publisher to generate positive margins from both sides of the market, for certain content categories.
\end{abstract}

Key words: Media, Advertising, Discrete Choice Model, Two-Sided Markets

JEL classification: L11, L52, L82, C33

We thank Le Groupe La Poste for providing us with the data and their generous help in using them. We are grateful for the invaluable comments of François Boldron, Claire Borsenberger, Denis Joram and Bernard Roy from the Department of Regulation, Institutional and European Affairs at Le Groupe La Poste and our colleague Sylvain Bourjade. The views presented here are those of the authors and do not necessarily represent the views of Le Groupe La Poste. All errors are our own.

Corresponding author: Catherine Muller-Vibes, Toulouse Business School, catherine.muller@tbs-education.fr 


\section{Introduction}

As a typical illustration of the so-called two-sided platform business model, print media generates revenues from two distinct sources of demand emanating from readers and advertisers. Those sources of demand are, however, interdependent as follows: The size of the readership has an impact on a periodical's attractiveness to advertisers, and the quantity of advertising influences readers' willingness to purchase a specific periodical. Most advertising-supported media earn much of their revenues from advertisers; to what extent do viewers value advertising? These indirect network effects on both markets should be accurately identified by competing firms to make appropriate strategic and marketing decisions. Pricing decisions will include the elasticities of the responses on both sides of the market. Thanks to a comprehensive and unique dataset, our empirical analysis of the French print media market allows us to assess structurally the interactions between the two sides of the market and show that readers value advertisements more than they might admit, specifically in the entertainment magazine category. To our knowledge, this feature of the print media market has not yet been consistently investigated.

Revenues for the print media sector in France have been decreasing in the past decade (with a 5.3\% decrease in global revenue between 2012 and 2013) according to a study conducted by the French Ministry of Culture and Communication in 2013. This fall in global revenue is due to decreases in both publication sales and advertising insert revenues, the latter showing the sharper decline. ${ }^{1}$ Unsurprisingly, those structural changes have strongly motivated industry stakeholders and policy makers to thoroughly understand the features of this market. Failing to consider the two-sidedness of the market precludes identifying the correct substitution patterns between the different periodicals and leads to biased strategic decisions, particularly regarding prices and quantities. The existence of a reciprocal effect between readership size and amount of advertising has partially been addressed empirically; however, we go further here in estimating it structurally and in distinguishing this effect between newspapers and magazines.

This tradeoff between content and advertising has seldom been addressed in the literature. Only a few empirical studies have been published, and they focus only on one of the two interactions between the size of the readership and the amount of advertising, omitting to evaluate their reciprocal effect. In a marketing framework, Godes et al. (2009) analyze theoretically how the two-sided competition affects firms' pricing decisions. They show that press editors may charge higher content prices in a duopoly than in a monopoly, in contrast to the standard one-sided market results. Depken and Wilson (2004) empirically examine whether consumers value advertising by estimating the demand for US magazine subscriptions in the nineteen-nineties. They find that relatively more advertising content reduces both the quantity and prices of magazine subscriptions. Argentesi and Ivaldi (2005) estimate the demand for eight French magazines from 1996 to 2001 instrumenting for advertising, and they show that neglecting the effect of advertising may have a significant impact on the estimated elasticities. Kaiser and Wright (2006) estimate a model of competition in a two-sided market where German magazines compete in a Hotelling fashion and find that the reader market is subsidized by advertising.

Note that only two empirical studies find a positive effect of advertising the readership: Filistrucchi, Klein and Michielsen (2011) on the Dutch newspapers market and Kaiser and Song (2009) on the magazine segment.

Assessing the effects of concentration on a market and identifying pricing strategies are areas of focus 
in the empirical literature on two-sided markets. Argentesi and Filistrucchi (2007) estimate a supply and demand model characterizing the Italian newspaper industry and find evidence of joint profit maximization in the cover prices of newspapers and competition on the advertising market but assuming a one-way externality only. Filistrucchi, Klein and Michielsen (2011) use a similar approach to estimate price elasticities, network effects and marginal costs in the Dutch newspaper industry and find that the effects of a merger on prices are weaker when the two-sidedness of the market is considered.

Rochet and Tirole (2003, 2005), Caillaud and Jullien (2003) and Armstrong (2006) emphasize that the accurate modeling of interactions between the two sides of a market is essential for an unbiased evaluation of a relevant market and market power, as prices may often significantly differ from their respective marginal costs because one side of the market subsidizes the other side.

Here, we contribute to the literature by significantly improving both the specification and estimation of demand functions for the two-sided market under analysis, namely the French print press industry comprising 282 newspapers and magazines over the period 2004-2006. To our knowledge this is the only study derived from such a comprehensive dataset gathering a high number of titles, both of newspapers and magazines, with information on sales at newsstand and by subscription. The results of our analysis then accurately reflect the substitution patterns of this industry. Our contribution is threefold. First, we structurally allow for the two externalities that can potentially characterize the working of this market, specifically the effect of the periodicals' circulation on advertising and readers' perception of the advertising. Second, we simultaneously estimate the demands of readership and advertising. Third, we allow the data to determine the signs of the effects of these externalities according to the editorial lines of associated periodicals. Hence, we allow readers not to present the same sensitivity or reactivity to advertising when reading a periodical with news-related content or an entertainment magazine

The paper is structured as follows. In the next section, we describe in detail the database that we use in our estimations. In the third section, we present the model that we believe is well suited for analyzing the French print media market, and then we describe the empirical implementation and results before concluding.

\section{The data}

The data are obtained from the Office de Justification de la Diffusion (OJD), which maintains records on the French print press exhaustively covering all French newspapers and magazines. Although there are missing data, we can build a large and meaningful sub-sample of 282 titles comprising all the types of periodicals in terms of content and frequency, from daily newspapers to quarterly specialized magazines, present on the market over the years 2004-2006. The dataset provides annual information on subscriptions, newsstand sales, subscription fees, cover prices, publication characteristics, number of yearly issues, number of advertising pages and total revenues from advertising. ${ }^{2}$

Data on online media are not available for this analysis; however, our model and estimation are robust to this lack of information because the period under analysis corresponds to the very early days of online press development and this population of potential consumers is included in our analysis as the so-called outside good

\footnotetext{
${ }^{2}$ For subscription fees to match cover prices, we divide the available annual subscription fee by the number of annual issues.
} 
(described in more detail in the model section). This outside alternative captures potential readers, and we approximate its size using i) data from the French national statistics institute (INSEE) on the distribution of the French population by age, ii) a study conducted by the Audience et Etudes de Presse Magazine (AEPM) for the years 2006 and 2007, which estimates that $97.2 \%$ of the French population above 15 years old reads at least one magazine per year and that $47 \%$ regularly reads daily newspapers.

\section{The model}

The crucial element in modeling both types of demand faced by press editors is the impact of the readership on the level of advertising demand and the intensity of advertising on the number of periodicals sold. This two-sided dimension of the market is captured by a two-way interaction between both the demand expressions. We assume that, together with price and periodical characteristics, the number of advertising pages has an impact on the number of issues sold and that the popularity of a periodical is itself a driver of advertising slot purchases.

\subsection{Reader demand}

We consider here the choices made by consumers willing to read one of the different periodicals. These choices are made in terms of topic and mode of purchase (at newsstands or by subscription). The different periodicals are characterized by quality characteristics and price. Consumers first choose the type of periodical that they want to buy, which can be classified into publications with news-related content or entertainmentoriented publications. Periodicals belonging to the categories "News Magazines", "Daily" and "Regional Weekly" are classified in the news group, whereas all the other categories fall into the entertainment group. ${ }^{3}$ Second, consumers choose the periodical, and, third, the purchase mode: at a newsstand or by subscription. We assume the existence of an outside alternative, which is characterized by reading a periodical without buying it, as other family members or employees of a firm would do. The outside alternative then captures the potential additional demand for newspapers and magazines. Hence, the total market size is defined as the total readers, which are interpreted as the total potential buyers of the periodicals. A choice for a consumer, then, is a combination of reading topic, publication title and purchase mode. The consumer choice structure is depicted in Figure 1.

\footnotetext{
${ }^{3}$ Alternative classifications were tested but brought meaningless results.
} 
Figure 1. Consumer Choice Tree

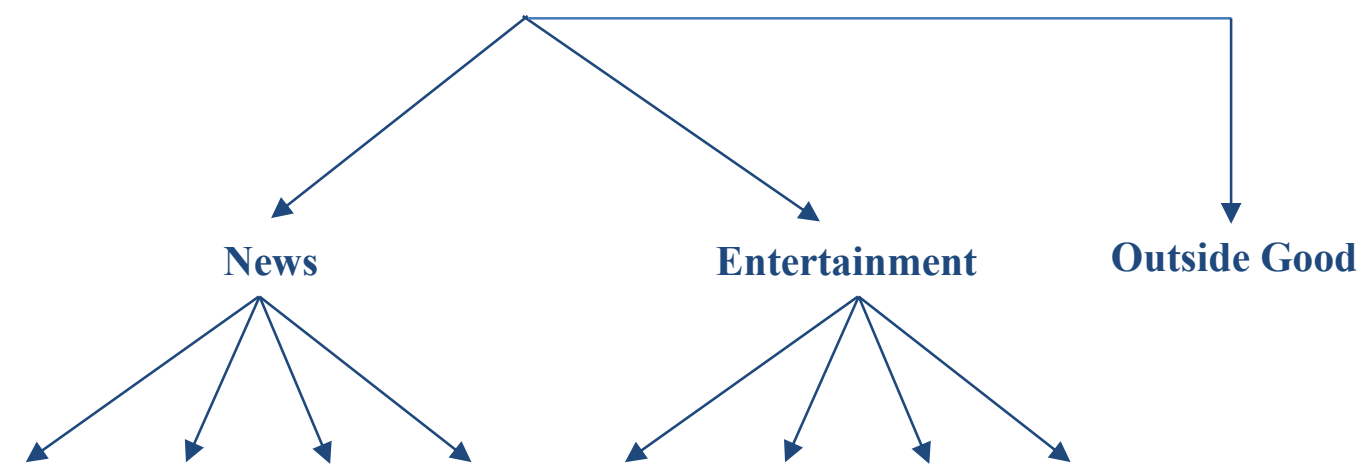

Newspaper 1 Newspaper 2 Newspaper 3

Magazine 1 Magazine 2 Magazine $3 \ldots$
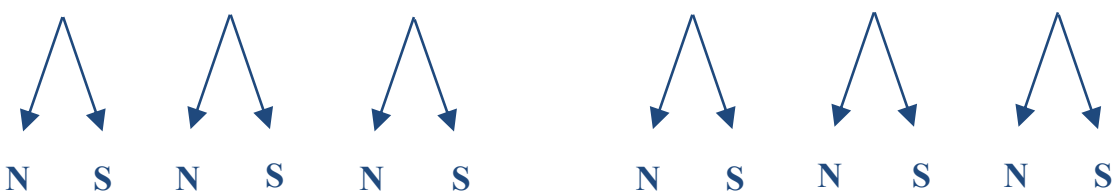

In the first step of choosing a periodical, a consumer chooses one of the two reading categories or the outside good $O G$. The two distinct categories are News and Entertainment. Let $g$ be the choice made by the consumer in the choice set $G=\{$ News, Entertainment, $O G\}$. In a second stage, the consumer chooses among the periodical titles available in the category that he chose in the first place. Let $h$ be the publication title selected by the consumer in the set $H_{g}$ of periodicals conditional on the choice $g$ in the first stage. Finally, in the third stage, the consumer chooses one way to purchase the periodical, namely at newsstand or by subscription. Let $k$ be the choice made by the consumer in the set $K_{h g}=\{$ Newsstand,Subscription $\}$ conditional on the choice $\{h, g\}$. In our case, both purchasing options are available for all the periodicals.

Please note in our French dataset of publications, we do not have information distinguishing single or grouped purchases, which prevents us from conducting a robustness check for a multi-homing behavior of readers. Kaiser and Wright (2006) on the German magazine market perform that comparison and find similar results. They also argue that the most adequate specification should be one of a partial-homing model for the publications market, as some readers purchase only one periodical, when others purchase more than one. They emphasize that such partial-homing models do not exist in the literature because of their complexity, and that one needs to choose to apply either a single-homing model or a multi-homing model. They underline that a single-homing behavior characterizes $80 \%$ of the magazines subscriptions in Germany. We are aware of the limitation of this specification, but we also believe for our data aggregation level (national and yearly), the distinction between single and multi-homing is not as applicable as at the consumer purchase level.

The readers' market demand is derived from a general class of discrete choice models of consumer behavior. For this discrete choice structure, an appropriate specification for an empirical analysis is the nested logit model. Applying the methodology developed by Berry (1994) and considering observed market shares as measures of choice probabilities, the demand specification associated with alternative $k$ is given by Equation (1) 
below. ${ }^{4}$ Ivaldi and Verboven (2005) provide the detailed algebra for this equation.

$\ln \left(\frac{q_{k}}{N-\sum_{k \in K_{h g}, \forall h, g} q_{k}}\right)=x_{k} \beta-\alpha p_{k}+\beta^{\text {news }} q_{h}^{a} D_{\text {news }}+\beta^{\text {ent }} q_{h}^{a} D_{\text {ent }}+\sigma_{K} \ln \frac{q_{k}}{Q_{h g}}+\sigma_{H} \ln \frac{Q_{h g}}{Q_{g}}+\xi_{k}$

where $q_{k}$ is the quantity of periodical $h$ sold at newsstands or by subscription; $Q_{h g}$ and $Q_{g}$ are, respectively, the total quantity of periodicals belonging to nest $\{h, g\}$ and nest $g$ sold on the market. $q_{h}^{a}$ is the number of advertising pages in publication $h$.

The deterministic part of the demand function is specified as a linear combination of available exogenous variables. Let $x$ be this set of exogenous variables. $\beta$ is a vector of parameters to be estimated. $\xi_{k}$ is the random component of the mean utility level common to all consumers. $p_{k}$ is the price of the selected product, and $\alpha$ is the sensitivity of utility to price.

We also assume that advertising has a different impact on readers depending on the type of periodical in which it is published. Specifically, we distinguish the effect of advertising in news-related periodicals from the effect of advertising in entertainment-oriented magazines. We multiply the variable on advertising quantity, $q_{h}^{a}$, by two different dummy variables, $D_{\text {news }}$ and $D_{\text {ent }}$, describing whether the publication belongs to the news or the entertainment category, respectively. $\beta^{\text {news }}$ and $\beta^{\text {ent }}$ are parameters to be estimated; they measure the impact of the quantity of advertising on reader demand relative to the periodical reading category.

We adopt here a nested logit specification for random preferences. In this framework, products within the same group are closer substitutes than products from different groups. The parameters $\sigma_{H}$ and $\sigma_{K}$, to be estimated, give a measure of the degree of correlation of alternatives belonging to the same group $g$ and subgroup $h$, respectively. These parameters must lie between 0 and 1 . The higher that $\sigma_{H}$ is, the higher the correlation between alternatives of the same group is, and the higher that $\sigma_{K}$ is, the higher the correlation between alternatives of the same subgroup is.

The set of variables $x$ comprises a first dummy variable $D_{\text {reg }}$ distinguishing between periodicals with regional and national content. A second variable $D_{\text {delivery }}$ takes the value 1 if the periodical is distributed by a different carrier than La Poste for the subscriptions. $D_{\text {freq }}$ takes the value 1 if the periodical's frequency is twice monthly, monthly, bi-monthly or quarterly, and 0 otherwise. ${ }^{5}$ The fourth dummy variable $D_{T V}$ aims at isolating the category of television guides, which presents the distinctive feature of being closely related to another very popular medium in France, namely television. This aspect is interpreted as another source of reader demand increase. We also include a variable $D_{\text {subs }}$ controlling for publications purchased at newsstands or by

\footnotetext{
${ }^{4}$ Time subscripts have been omitted for simplicity of exposure and without loss of generality.
} 
subscription to capture the difference in quality between these two purchase options, and a trend.

Please note that we take customer heterogeneity into account here through the differentiation of products, which incorporates consumers' preferences. To characterize the periodicals, we account for the main differentiating criteria for these products (described above). The nested logit model is then legitimate, compared to a random coefficient model, according to Grigolon and Verboven (2015).

\subsection{Advertiser demand}

Following Ivaldi and Zhang (2017), we consider a representative advertiser whose expected revenue from an advertising insert in publication $h$ is denoted by $r_{h}$ and such that $r_{h}=\tau_{h} c_{h} . c_{h}$ is the advertisers' willingness to pay for reaching publication $h$ 's readership, and $\tau_{h}$ is a scaling factor at least larger than 1 (we assume that the willingness to pay of the advertisers represents only a fraction of their expected benefit from advertising. Let $p_{h}^{a}$ be the average price of an ad insert in publication $h$. We specify the representative advertiser's profit function as:

$$
\Pi^{a}=\sum_{h=1}^{H}\left(\tau_{h} c_{h}-p_{h}^{a} q_{h}^{a}\right)
$$

On the advertising market, publishers and advertisers agree on a $c_{h}$ based on the size of the readership for publication $h, q_{h}$, the number of advertising pages in periodical $h, q_{h}^{a}$, and some observed characteristics of publication $h, z_{h}$. We thus specify the market-determined $c_{h}$ using a Cobb-Douglas form:

$$
c_{h}=q_{h}^{\mu^{r e a d}} q_{h}^{a \mu^{a}} z_{h}{ }^{\mu}
$$

where $q_{h}$ is the quantity of readers buying periodical $h$ and we control for a set of variables called $z_{h}$.

Replacing the expression into the profit equation above and maximizing $\Pi^{a}$ over $q_{h}^{a}$, we get the following demand function for advertising in logarithmic form, to be estimated:

$$
\ln \left(q_{h}^{a}\right)=\gamma_{0}-\gamma \ln \left(p_{h}^{a}\right)+\gamma^{\text {read }} \ln \left(q_{h}\right)+\gamma^{z} \ln \left(z_{h}\right)+\xi_{h}^{a}
$$

where $\gamma_{0}, \gamma, \gamma^{z}, \gamma^{\text {read }}$ are parameters to be estimated. $\gamma$ is advertisers' sensitivity to price, and $\gamma^{\text {read }}$ measures the effect of publication circulation on advertising demand. $\xi_{h}^{a}$ is a random term reflecting the effect of unobserved characteristics of advertising in periodical $h$ on its profit.

\footnotetext{
${ }^{5}$ Remaining frequencies are daily, weekly and three times weekly.
} 


\section{Empirical implementation}

Due to the two-sided aspect of this press market, both demand equations (1) and (4) are estimated simultaneously. To overcome the issue of endogeneity of prices in our specification, we apply an instrumentalvariable procedure, namely the Generalized Method of Moments. We use the MODEL procedure of the SAS software.

We use two different sets of instruments for the two demand equations, which both consist in "BLP instruments" (characteristics of competing products) and other exogenous characteristics of the periodicals. For the readers' demand function, we use the average number of pages in one issue of competing titles in the same group $G$, the lag of the average price (at newsstand and by subscription) of competing publications in the same group $G$, a dummy for low frequency publications (monthly to quarterly), a dummy for the two categories almost exclusively purchased at newsstand (>90\%): « Men » and « Tabloids » and a dummy for daily publications. The set of instruments used for the advertisers' demand equation is the lag of the average price for an ad insert of competing publications, the yearly number of issues and a dummy for high frequency publications (daily to triweekly). A set of instruments to be statistically validated needs to satisfy the following two requirements: being correlated with the endogenous explanatory variables and being exogenous (uncorrelated with the structural error). First, the returned first stage R-squares are between 0.52 and 0.98 for our right hand side endogenous variables, confirming the explanatory power of our instruments, and suggesting that weak identification should not be a problem here. Second, we perform the appropriate Hansen $J$ test for overidentifying restrictions to check whether the instruments are uncorrelated with the structural error term. The $p$-value for this test at the $5 \%$ level is equal to $11 \%$ so we cannot reject the null hypothesis of our instruments being uncorrelated with the error term, validating our set of instruments for the estimation of our model.

\subsection{Estimation results}

The results of the estimation of demand equations (1) and (4) are presented in Table 1. First, we note that all the parameters are significantly different from 0 at a five-percent level. Second, the parameters of interest have the right signs and meaningful magnitudes. As expected, parameters $\alpha$ and $\gamma$ are positive, indicating that prices have negative effects on both demands according to our specification. Parameters $\sigma_{H}$ and $\sigma_{K}$, which capture the correlations of alternatives between the groups and subgroups, respectively, are greater than zero and smaller than one, as required by the nested logit model. ${ }^{6}$ Moreover, the parameter $\sigma_{K}$ is greater than the parameter $\sigma_{H}$, which means that substitution is stronger between alternatives from the same subgroup than alternatives from the same group. This finding is intuitive and required by the model to be consistent with utility maximization.

In this two-sided market framework, we note the significant interaction between the two demand equations, captured by the parameters $\beta^{\text {news }}, \beta^{\text {ent }}$ and $\gamma^{\text {read }}$. We first find that the effect of advertising on reader demand depends on the periodical's reading category. $\beta^{\text {news }}$ and $\beta^{\text {ent }}$ being, respectively, negative and positive, we see that advertising is a quality-improving characteristic of entertainment magazines, whereas it is 
not valued positively by consumers when published in a newspaper. This finding is consistent with our intuition. Newspapers promise very broad content on current events; readers want to access this information immediately and not to be disturbed by inserted advertisements. However, consumers purchase magazines for specific content related to their own interests. In each magazine, advertisements are related to the topic in question and are then positively valued by the readers. We also find that the volume of demand emanating from press buyers has a positive effect on advertising demand, which is an expected result, indicating that the more popular the periodical, the more attractive it is to advertisers to reach more potential clients for the products that they are advertising. To our knowledge, this result is new in the literature, specifically when obtained by estimating a structural model for these two effects. ${ }^{7}$

We progress further in understanding consumer behavior by computing readers' elasticity with respect to the quantity of advertising in a magazine or a newspaper. We estimate the following values:

- For an entertainment magazine, the average elasticity is 1.47 , with a variance of 0.99 .

- $\quad$ For a newspaper, the average elasticity is -0.7 , with a variance of $0.72 .^{8}$

For entertainment magazines, advertising elasticity is higher than one with a relatively small variance, confirming readers' sensitivity to advertising for this content category. For news related periodicals this elasticity is lower than one but with a higher variance, therefore indicating different readers' sensitivities to advertising among newspapers titles. The most crucial finding here is that these elasticities present opposite signs, conveying opposite reader preferences with respect to advertising depending on the publication category. When allocating content and publicity pages, an editor should consider the impact of adding more inserts to an issue, which is accurately measured by this demand elasticity in a model allowing for the reciprocal interaction between reader and advertiser demand. These results are further discussed at the end of the next section.

The other parameters of the model identify the characteristics of a periodical that are valued by readers and advertisers. In the reader demand, we see that nationally distributed publications are preferred to regional ones and that television guides are highly valued by readers. Distribution by a private carrier, lower frequencies than twice monthly and sales at newsstands are also preferred. The negative trend captures the observed fading interest of readers in print media. On the advertisers' side of the market, once the effect of press buyers' demand is considered, regional titles are not favored by the advertisers, whereas periodicals with lower frequencies generally are. The average volume of pages in a periodical is also positively valued by advertisers. This finding corroborates our previous intuition; advertisers know that advertisements are more likely to reach customers who read magazines than those who read newspapers. These consumers will probably be more attentive to advertisements that are directly related to their topics of interest.

\footnotetext{
${ }^{6}$ Please note that these parameters are not constrained in the estimation.

${ }^{7}$ Note that a similar model capturing only the effect of readership on advertising demand, which is often studied in the literature, leads to poor statistical quality of estimation on our sample.
} 
Table 1. Estimation Results

\begin{tabular}{lccc}
\hline \hline \multicolumn{4}{c}{ Reader demand equation } \\
\hline \hline Variable & Parameter & $\begin{array}{c}\text { Parameter } \\
\text { estimate }\end{array}$ & t-ratio \\
\hline$p_{k}$ & $\alpha$ & 0.103 & $3.60^{* * * *}$ \\
$\ln \frac{q_{k}}{Q_{h g}}$ & $\sigma_{K}$ & 0.712 & $5.04^{* * * *}$ \\
$\ln \frac{Q_{h g}}{Q_{g}}$ & $\sigma_{H}$ & 0.670 & $11.230^{* * *}$ \\
$q_{h}^{a} D_{\text {news }}$ & $\beta^{\text {news }}$ & -0.013 & $-3.76^{* * *}$ \\
$q_{h}^{a} D_{\text {ent }}$ & $\beta^{\text {ent }}$ & 0.011 & $7.346^{* * *}$ \\
$D_{\text {reg }}$ & $\beta^{\text {reg }}$ & -0.892 & $-12.23^{* * *}$ \\
$D_{\text {delivery }}$ & $\beta^{\text {delivery }}$ & 0.312 & $5.21^{* * *}$ \\
$D_{T V}$ & $\beta^{T V}$ & 0.531 & $5.10^{* * *}$ \\
$D_{\text {subs }}$ & $\beta^{\text {subs }}$ & 0.168 & $2.63^{* *}$ \\
Trend & $\beta^{\text {trend }}$ & -0.113 & $-7.77^{* * *}$ \\
& & &
\end{tabular}

Observations

\begin{tabular}{lccc}
\hline \hline \multicolumn{4}{c}{ Advertiser demand equation } \\
\hline \hline Variable & Parameter & $\begin{array}{c}\text { Parameter } \\
\text { estimate }\end{array}$ & t-ratio \\
\hline $\ln \left(p_{h}^{a}\right)$ & $\gamma$ & 2.895 & $4.56^{* * *}$ \\
$\ln \left(q_{h}\right)$ & $\gamma^{\text {read }}$ & 1.504 & $3.55^{* * *}$ \\
$D_{\text {freq }}$ & $\gamma^{\text {freq }}$ & 0.412 & $2.45^{* *}$ \\
$\ln ($ Pages $)$ & $\gamma^{\text {pages }}$ & 0.718 & $5.99^{* * *}$ \\
$D_{\text {reg }}$ & $\gamma^{\text {reg }}$ & 1.264 & $2.01^{* *}$ \\
Trend & $\gamma^{\text {trend }}$ & 0.113 & $2.08^{* *}$ \\
\hline “***” denotes significance at the 1\% level, and “**”denotes significance at the 5\% level.
\end{tabular}

\subsection{Price elasticities estimates}

Determining the price of a product or service is one of the most difficult tasks that a marketer must perform. Understanding the factors that make the demand elastic or inelastic is crucial for better designing

\footnotetext{
${ }^{8}$ Elasticity measures per periodical are available upon request.
} 
pricing and differentiation strategies in a fast-evolving competitive environment.

Own-price-elasticities can be computed from the estimation of equations (1) and (4). The box-plots displayed in Figure 2 represent the distributions of own-price elasticities for the readers' demand, averaged over time and grouped by reading category. Own-price elasticities measure the percentage variation of demand for a periodical following a one-percent change in price for that periodical.

Figure 2. Distribution of Own-Price Elasticities across Reading Categories

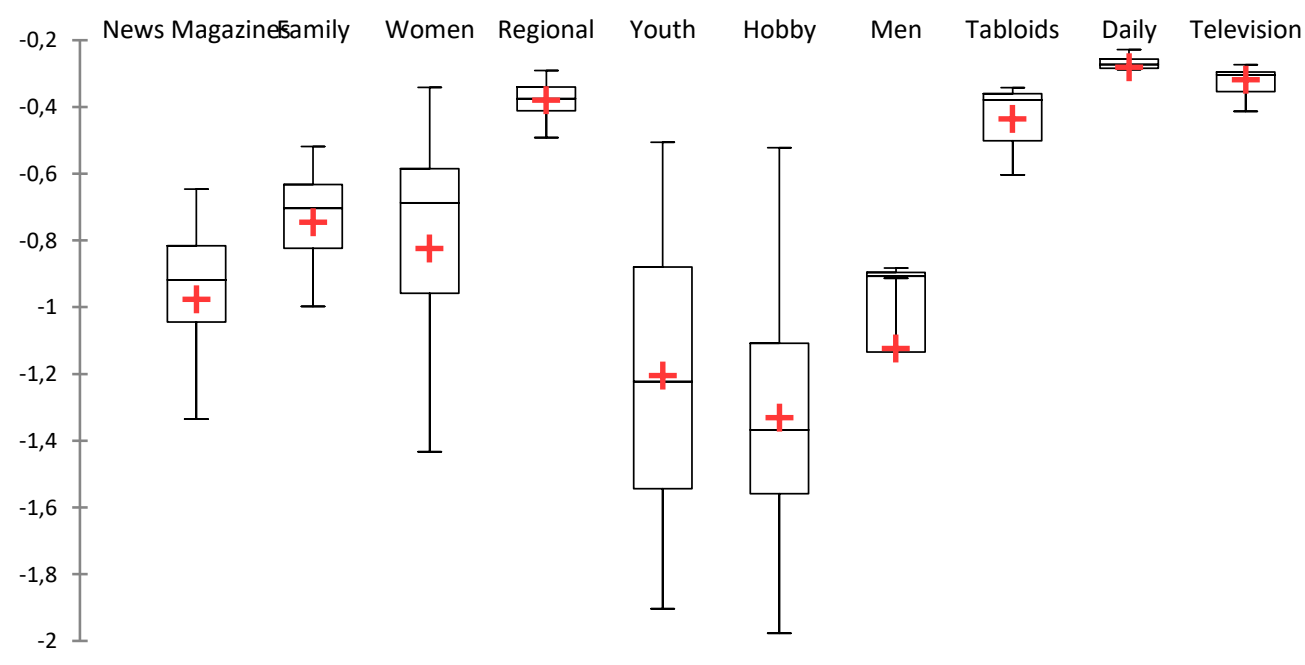

Red crosses on the graphs correspond to the means. Periodical categories for which demand is more elastic with respect to price are "News Magazines", "Youth", "Hobby" and "Men". We also observe the highest variance for these elasticities. These results are in line with the findings of Argentesi and Ivaldi (2005) on the demand analysis of a sample of French magazines. They indicate that substitutions between magazines from these categories are higher after a price increase than they are for the "Daily" and "Television" categories for example. Readers are more likely to switch to a different entertainment magazine after a price increase than from a newspaper or television guide. We also compute cross-price elasticities ${ }^{9}$, and they decrease as periodicals become less substitutable as defined in our nested logit model. This result suggests that advertisers who would choose categories exhibiting low and concentrated demand elasticities would benefit from a more regular audience for their long-lasting advertising campaigns.

On the advertisers' side, own-price elasticity of demand is equal to 2.9 on average with a standard error of 0.6 which is a sign that the advertising market is competitive. By simultaneously interpreting these elasticities, we could say first that advertisers needing to target a very specific category of the audience (such as young readers) would choose its corresponding periodicals and would trade off the size of the readership for these periodicals and the advertising insert price changes. Second, advertising campaigns aimed at reaching a broader spectrum of the population should, of course, focus on popular titles but also on the titles for which demand is inelastic and therefore more loyal to ensure a more consistent impact of their advertising campaign.

To summarize our findings, we can say that the positive readers' demand elasticity with respect to advertising in entertainment magazines creates the incentive for editors to print more ads in their periodicals, and 
to benefit from increased sales on both sides of the market. Yet it should be noted that the regulation in France does not allow more than two thirds of a publication's pages to be dedicated to advertising. We also find that for magazines with low price demand elasticity on the readers' side, editors' revenues could be improved by increasing prices. And for these magazines also, advertisers are likely to value their regular audience. These combined effects should allow a publisher to generate positive margins from both sides of the market, for these content categories. These results differ from the ones summarized in Sriram et al. (2015), where the author underlines that consumer market is subsidized by advertising revenue. We trust our rich dataset and estimation allow us to identify more specific substitutions and interactions in this two-sided-market, and thus lead to more precise conclusions for some categories of magazines.

\section{Conclusion}

In this paper, we exploit a database of 282 French periodicals over the period 2004 to 2006 to empirically measure the reciprocal interaction between the two sources of revenues in the print press industry, namely readership and advertising. We structurally model the two demand functions by allowing for the presence of two-way network externalities. We estimate the demand functions simultaneously and control for potential endogeneity of prices by using an instrumental variables technique. We allow the data to show that readers have a taste for advertising in magazines, whereas they would rather enjoy less advertising inserts in a newspaper. Price elasticities for readership and advertising demand are also computed for the same purpose. This study statistically proves that not considering the two-sidedness of the market in the print media industry and the periodical content may lead to unreliable measures of consumers' sensitivities to advertising, price and other characteristics that they value.

Future research could explore a model in which editors' strategic behavior is also taken into account to better assess competition effects and substitutability between periodicals. This research would add significant complexity to an estimation of a simultaneous equilibrium model and would require some high-quality additional data. We would also like to progress further by introducing online media activity and better investigating how the numerical versions of periodicals have modified the market mechanisms in recent years. We believe that our results are transposable to the digital media market.

\footnotetext{
${ }^{9}$ Available upon request.
} 


\section{References}

Argentesi, E., \& Filistrucchi, L. (2007). Estimating market power in a two-sided market: The case of newspapers. Journal of Applied Econometrics, 22(7), 1247-1266.

Argentesi, E., \& Ivaldi, M. (2005). Market Definition in Printed Media Industry: Theory and Practice. IDEI Working Paper, $n .376$.

Armstrong, M. (2006). Competition in two-sided markets. The RAND Journal of Economics, 37(3), 668-691.

Berry, S. T. (1994). Estimating discrete-choice models of product differentiation. The RAND Journal of Economics, 242-262.

Caillaud, B., \& Jullien, B. (2003). Chicken \& egg: Competition among intermediation service providers. RAND journal of Economics, 309-328.

Depken II, C. A., \& Wilson, D. P. (2004). Is advertising a good or a bad? Evidence from US magazine subscriptions. The Journal of Business, 77(S2), S61-S80.

Dewenter, R. (2003). The economics of media markets. University FAF Economics Discussion Paper, (10).

Filistrucchi, L., Klein, T. J., \& Michielsen, T. O. (2012). Assessing unilateral merger effects in a two-sided market: An application to the Dutch daily newspaper market. Journal of Competition Law and Economics, 8(2), 297-329.

Godes, D., Ofek, E., \& Sarvary, M. (2009). Content vs. advertising: The impact of competition on media firm strategy. Marketing Science, 28(1), 20-35.

Grigolon, L., \& Verboven, F. (2014). Nested logit or random coefficients logit? A comparison of alternative discrete choice models of product differentiation. Review of Economics and Statistics, 96(5), 916-935.

Ivaldi, M., \& Verboven, F. (2005). Quantifying the effects from horizontal mergers in European competition policy. International Journal of Industrial Organization, 23(9), 669-691.

Ivaldi, M., \& Zhang, J. (2017). Advertising Competition in the Free-to-Air TV Broadcasting Industry. TSE Working Paper.

Kaiser, U., \& Song, M. (2009). Do media consumers really dislike advertising? An empirical assessment of the role of advertising in print media markets. International Journal of Industrial Organization, 27(2), $292-301$.

Kaiser, U., \& Wright, J. (2006). Price structure in two-sided markets: Evidence from the magazine industry. International Journal of Industrial Organization,24(1), 1-28.

Rochet, J. C., \& Tirole, J. (2003). Platform competition in two-sided markets. Journal of the European Economic Association, 1(4), 990-1029.

Rochet, J. C., \& Tirole, J. (2005). Competition Policy in Two-Sided Markets. Advances in the Economics of Competition Law, MIT Press, Cambridge, MA, USA.

Sriram, S., Manchanda, P., Bravo, M. E., Chu, J., Ma, L., Song, M., ... \& Subramanian, U. (2015). Platforms: a multiplicity of research opportunities. Marketing Letters, 26(2), 141-152.

Spitz, B., Frappat, B., Puyfontaine, A., Patino, B. \& Dufour, F. (2009). Etats généraux de la presse écrite, livre vert. http://www.ladocumentationfrancaise.fr/rapportspublics/094000017/index.shtml 